Methods Cross-sectional study was conducted among undergraduate students of Gulf Medical University using a self administered questionnaire as research instrument for data collection. The demographic data and the wheezing and bronchial asthma characteristics, family history was collected and analyzed using SPSS version 19. Chi square test used to test association between variables.

Results A total of 255 students were included; of which 71(27.8\%) had wheezing (Females were $66 \%$ and males 34\%); Wheezing was more prevalent among African(18\%) and Pakistani(16\%) nationals. Of 71 students, 41(57.7\%) students experienced 1-3 episodes in last one year, 13(18.3\%) had episodes limiting their speech. Exercise induced wheezing was experienced by $62(24.3 \%)$ participants. The wheezing episodes were most frequent in January and March $(p<0.05)$. Positive family history of wheezing was reported by $29(40.8 \%)$ students. $40.8 \%$ students reported that wheezing interfered with their daily and extra-curricular activities. Of the total, $31(12.2 \%)$ students gave history of bronchial asthma of which 16 $(50 \%)$ had a positive family history of bronchial asthma.

Conclusions Overall higher proportion of students had wheezing symptoms especially among the African students. Strong family history was also noted both wheezing and bronchial asthma.

\section{A STUDY OF INFLUENCE OF RELATIONSHIP ON LIFE STYLE AND MENTAL HEALTH OF ADOLESCENT}

doi:10.1136/archdischild-2012-302724.0447

\section{Sharma. NPSP, WHO, Bikaner, India}

Adolescent period when teen having impact of broken relationship, which affects teens ability to function in life.

Aims \& objectives:

1. To study influence of relationship and explore possible causes and solutions to this problem

2. To highlight the importance of impact of relationship on life style and mental health of teens.

Material and method Study done in two Private coaching institute.

250 children of age group 15 to 20 years from two renowned institutes with their parents were interviewed with the help of a pre-set questionnaire. The questions were aimed at knowing the life style and impact of relationship on these children. From this information various problems in each child were identified and then analyzed.

Observations Total number of children was 250, 140 were girls and rest 110 boys. age from 15 to 20 years. 30\% of joint family, $45 \%$ nuclear family and $25 \%, 10 \%$ broken home Impact on mental health $50 \%$, Social withdrawal $25 \%$ Refusal to go to school $25 \%$ Poor study behavior and skills $10 \%$, disruptive behavior in classroom $12 \%$, school performance dropping $30 \%$, emotionally unstable behavior $10 \%, 20 \%$ Poor hygiene and grooming 15\% Involved in reckless activities $15 \%$ Using alcohol or other drugs 10\% Skipping school $10 \%$ violent behavior.

Conclusion The study clearly bring out that, there is strong influence $\&$ impact on the mental health and social development and life style of children Broken relationship having strong impact on mental health, this can be controlled timely by parents with trust and open communication and adolescent counseling.

\section{EXUBERANT BILATERAL INGUINAL LYMPHADENOPATHIES LESS TYPICAL MANIFESTATION OF CAT SCRATCH DISEASE}

doi:10.1136/archdischild-2012-302724.0448

${ }^{1} \mathrm{C}$ Patraquim, 'Ml Alves, ${ }^{1} \mathrm{~A}$ Antunes, 'T Pontes, 'S Martins, ${ }^{1,2} \mathrm{H}$ Antunes. ${ }^{1} \mathrm{Hospital}$ de Braga, Braga; ${ }^{2}$ Instituto de Ciências da Vida e da Saúde (ICVS), Escola de Ciências da Saúde da Universidade do Minho, Laboratório Associado ICVS/3B's, Braga/Guimarães, Portugal
Introduction Cat scratch disease(CSD) is caused by Bartonella henselae $(\mathrm{BH})$. Regional lymphadenopathy, located to the neck and/ or axilla, is the hallmark of CSD.

Case Description A 17-year-old girl presented with a painful right inguinal swelling for the past 3 weeks. Cited contact with small cats. Presented objectively bilateral inguinal lymph node swelling of hardelastic consistency, $2 \mathrm{~cm}$ diameter each, barely mobile, with overlying cutaneous inflammatory signs. There were no other palpable lymph nodes, hepatosplenomegaly, lower limbs' abrasions, or perineal abnormalities. C-reactive protein: $43.40 \mathrm{mg} / \mathrm{L}(\mathrm{N}:<2.9 \mathrm{mg} / \mathrm{L})$. Inguinal ultrasound suggested cellulite with reactive adenitis. The hypothesis of bacterial abscess or $\mathrm{BH}$ infection were most probable, however, other diagnoses were considered such as sexually transmitted disease, tuberculosis lymphadenitis or, eventually, lymphoma.

She was hospitalized with (i.v.)intravenous flucloxacillin. Abdominopelvic ultrasound, chest radiograph, and infectious serologies were normal. BH serology remained in progress during hospitalization. Elevation of erythrocyte sedimention rate $(75 \mathrm{~mm} / \mathrm{h})$ Tuberculin test was negative.

CSD was believed to be the most probable diagnosis, despite the aspect and location of lymphadenopathies, and i.v. clarithromycin was iniciated. Aspirative punction of the enlarged inguinal lymph nodes was performed, exteriorizing a purulent material. BH serology for IgM and IgG was positive. Cytology showed acute inflammatory process with suppuration, and microbiological test was sterile. Searching for bacterial DNA was positive for BH. A month later, she had right inguinal suppuration, and reduction of the lymph nodes size and tenderness.

Discussion The exuberant bilateral lymphadenopathies in less typical location were important confounding factors that led to complementary exams in an usually benign disease.

\section{PREVALENCE OF ALLERGY AMONG UNIVERSITY STUDENTS, AJMAN, UAE}

doi:10.1136/archdischild-2012-302724.0449

'LJ John, ${ }^{2} \mathrm{M}$ Arifulla, ${ }^{2} \mathrm{~J}$ Sreedharan. ${ }^{\text {PPharmacology; }}{ }^{2}$ Gulf Medical University, Ajman, United Arab Emirates

Background and Aims Urbanization and globalization in UAE has resulted in drastic environmental changes and increase in allergens present in the environment and also the prevalence of allergies. Hence, this study aimed to assess the prevalence of allergies among undergraduate students of Gulf Medical University.

Methods This cross-sectional survey was carried out among undergraduate students of Gulf Medical University. A self administered questionnaire was used as research instrument for data collection. The demographic data and allergy characteristics were collected and analyzed using SPSS version 19. Descriptive and analytic statistics were performed.

Results A total of 255 students were included (31\% males, 69\% females). The commonest allergies among university students were allergic rhinitis 120(47\%); allergic conjunctivitis 104(40.8\%), atopic dermatitis $89(34.9 \%)$ and bronchial asthma $31(12.2 \%)$ in the rank order. Positive family history of allergies was reported by $62(24.3 \%)$ for atopic dermatitis, 39(15.3\%) for allergic rhinitis and 33(12.9\%) for allergic conjunctivitis. The allergies were common among Pakistani and African nationals. Allergic rhinitis was also frequent among Emiratis. Allergies were common during months of January and December. Allergies associated with pollen, food and drug were less frequent. The distribution of allergies based on gender revealed female preponderance in all the types of allergy. Students with allergies reported interference with their daily activities, academic activities, social and extra-curricular activities.

Conclusions Overall higher prevalence of allergies noted among students especially the African and Pakistani students. Adequate 
preventive strategies should be initiated to curb this rising prevalence of allergies.

\section{WHAT DO CHILDREN WITH CONGENITAL HEART DISEASES DO WHEN THEY GROW UP}

doi:10.1136/archdischild-2012-302724.0450

SC Antoniadis, A Antoniadou, O Panagiotopoulos, E Milopteri, P Kleanthous, E Dionyssopoulou. Diagnostic Center for Pediatric Cardiology, Athínai, Greece

Background Advances in the diagnosis and interventional-surgical management of congenital heart diseases had as a result a considerable number of patients reaching the adolescent and young adult life.

Aims The aim of this study was to investigate the influence of the heart problem on the psychosocial-sexual and family life, careers and employment rate of patients with congenital heart disease.

Methods We studied according to a protocol with a closed questionnaire as well as the medical files of 441 children with congenital heart diseases.

Results From the 158 male patients aged $15-35$ years, 98(62\%) had simple heart lesions, 21(13.3\%) combinations of simple heart lesions and 39(24.7\%) complex heart problems, 93(59\%) had surgicalinterventional management. Between them 130(82.2\%) had no psychosocial-sexual problems related with age, whereas $13(8.3 \%)$ with complex heart lesions had not normal social and sexual life. From the 146 female patients aged $14-32$ years, 93(63.7\%) had simple lesions, $25(17.12 \%)$ combinations of simple heart lesions, and $28(19.17 \%)$ complex heart problems, $56(38.3 \%)$ had surgicalinterventional management. From them $136(93.15 \%)$ had no problems related with age whereas $10(6.85 \%)$ had, from the 23 pregnancies, $10(43.5 \%)$ operated mothers, $21(91.3 \%)$ babies born without problems. From the 114 patients studied for their careers and employment rate, 59(59.8\%) were high school graduates, $43(37.7 \%)$ university graduates, $9(7.9 \%)$ technical school graduates, $103(90.4 \%)$ were employed, 11(9.6\%) couldn't work because of the heart problem.

Conclusions Simple heart lesions and their combinations doesn't cause specific problems whereas complex heart diseases, especially if the result of the operation is not the expected one, cause.

\section{THE EFFECT OF PRETERM BIRTH ON ATTENTIONAL NETWORKS IN ADLULTS}

doi:10.1136/archdischild-2012-302724.0451

A von Muhlenen, N Baumann, D Wolke. University of Warwick, Coventry, UK

Background Preterm birth has been associated with an increased risk of cognitive, behavioral and psychiatric problems. In this study we examine how these problems are linked to specific attentional networks.

Methods Based on a sample from the Bavarian Longitudinal Study we compared a cohort of 97 survivors born very preterm $(<32$ weeks gestational age, GA) or very-low-birth-weight (VLBW < $1500 \mathrm{~g}$ ) in 1985/86 (mean birth weight, 1318g; mean GA at birth, 30.2 weeks; $52 \%$ male) with 109 controls from the same population in Bavaria (mean birth weight, 3414g; mean GA at birth, 39.7 weeks; $48 \%$ male). We looked at performance in the attention network test, developed by Fan et al. (2002, Journal of Cognitive Neuroscience), in three anatomically defined attentional networks: alerting, orienting and executive control.

Results The figure shows the RT index, representing efficiency for each attentional network. The VLBW group showed a large and highly significant deficit in the executive network and a smaller but significant deficit in the orienting network, but no deficit in the alerting network. Although RTs were generally longer for VLBW, the RT index result did not change when using RT ratio scores.

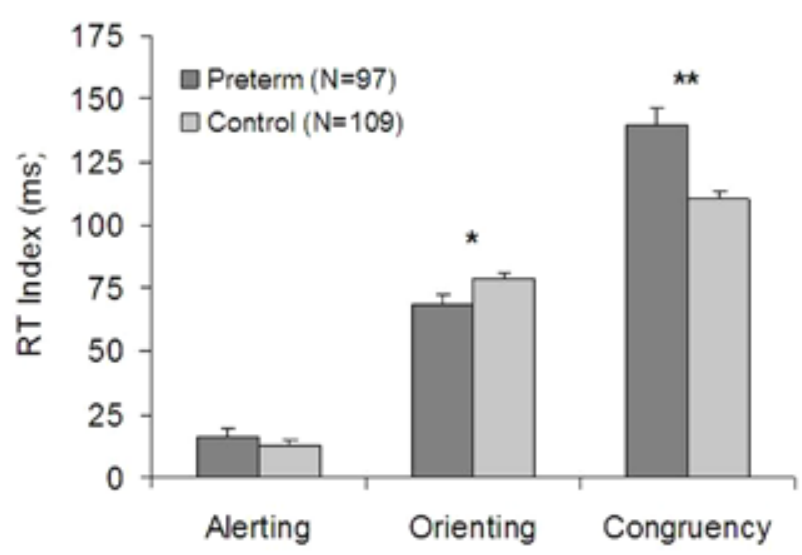

Attentional Network

Abstract 451 Figure 1 RT Index

Conclusions The results show that VLBW is linked to specific attentional deficiencies, especially those involving executive control. These results are discussed in the light of the literature on VLBW and attention.

\section{ASSOCIATIONS BETWEEN BEHAVIORAL AND SLEEPING DISORDERS IN PRETERM CHILDREN TO CATCH UP IN HEIGHT AND WEIGHT}

doi:10.1136/archdischild-2012-302724.0452

${ }^{1}$ E Deschman, ${ }^{1} \mathrm{M}$ Vanpee, ${ }^{1} \mathrm{~L}$ Legnevall, ${ }^{2} \mathrm{~A}$ Kistner. ${ }^{1}$ Inst of Women and Child Health; 2Inst of Molecular Medicine and Surgery, Stockholm, Sweden

Aim To study an association between sleeping (SP) and behavioural disorders (BP) in preterm children to catch-up growth and weight.

Design and Methods 105 children (8.5-10 years): 39 born preterm (<32 weeks), at term 30 born SGA and 36 born AGA (Controls) were studied. Eight Preterms were born SGA and 31 were born AGA. BP was defined as hyperactivity and concentration problems, SP as hard to fall asleep and wakening during night. Pearson Chi-Square or Student t-test were used.

Results The Preterm group displayed a higher incidence of SP $(23 \%$ vs $10 \%$ and $3 \% \mathrm{p}=0.026)$ and $\mathrm{BP}(38 \%$ vs $10 \%$ and $8 \%, \mathrm{p}=0.001)$.

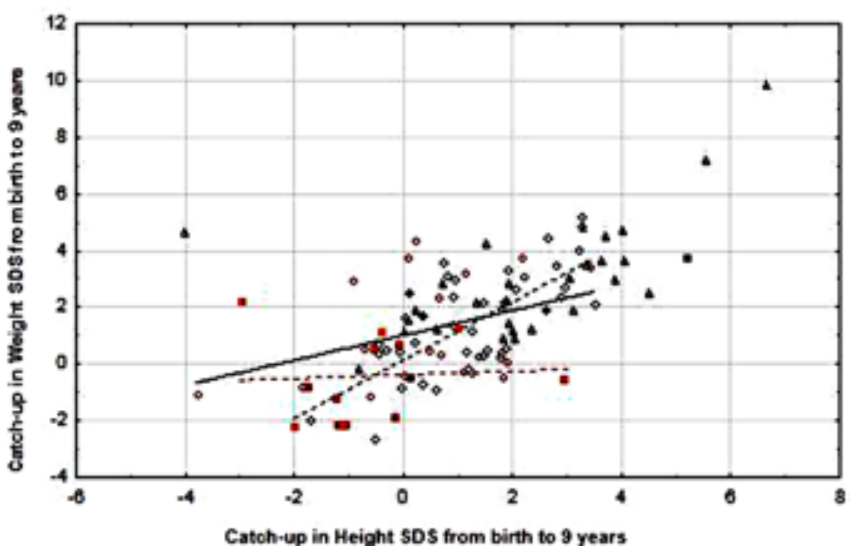

Abstract 452 Figure 1 BP in relation to catch up growth

o Preterm AGA no BP•Preterm AGA with BP $\diamond$ Control no BP 